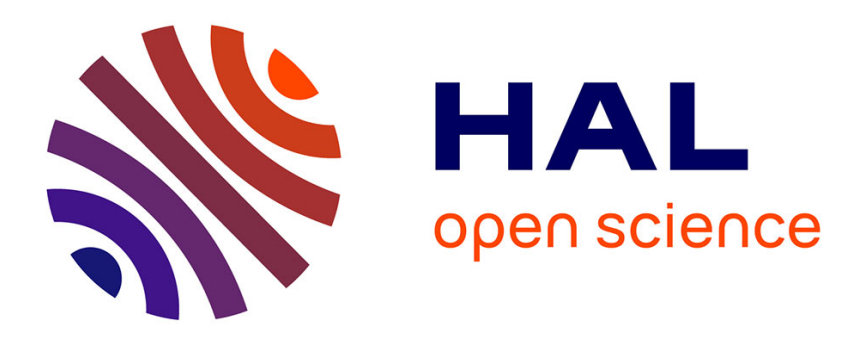

\title{
The first negative coefficients of symmetric square L-functions
}

Y.-K Lau, J.-y Liu, J Wu

\section{To cite this version:}

Y.-K Lau, J.-y Liu, J Wu. The first negative coefficients of symmetric square L-functions. Ramanujan Journal (The), 2012, 10.1007/s11139-011-9318-x . hal-01278389

\section{HAL Id: hal-01278389 \\ https://hal.science/hal-01278389}

Submitted on 24 Feb 2016

HAL is a multi-disciplinary open access archive for the deposit and dissemination of scientific research documents, whether they are published or not. The documents may come from teaching and research institutions in France or abroad, or from public or private research centers.
L'archive ouverte pluridisciplinaire HAL, est destinée au dépôt et à la diffusion de documents scientifiques de niveau recherche, publiés ou non, émanant des établissements d'enseignement et de recherche français ou étrangers, des laboratoires publics ou privés. 


\title{
THE FIRST NEGATIVE COEFFICIENTS OF SYMMETRIC SQUARE $L$-FUNCTIONS
}

\author{
Y.-K. LAU, J.-Y. LIU \& J. WU
}

\begin{abstract}
Let $n_{\mathrm{sym}^{2} f}$ be the greatest integer such that $\lambda_{\mathrm{sym}^{2} f}(n) \geqslant 0$ for all $n<n_{\mathrm{sym}^{2} f}$ and $(n, N)=1$, where $\lambda_{\mathrm{sym}^{2} f}(n)$ is the $n$th coefficient of the Dirichlet series representation of the symmetric square $L$-function $L\left(s, \mathrm{sym}^{2} f\right)$ associated to a primitive form $f$ of level $N$ and of weight $k$. In this paper we establish the subconvexity bound: $n_{\mathrm{sym}^{2} f} \ll\left(k^{3} N^{2}\right)^{40 / 113}$ where the implied constant is absolute.
\end{abstract}

\section{INTRODUCTION}

A classical question in analytic number theory concerns the least quadratic nonresidue, see for example, $[30,2,18,16]$ for some investigations. More importantly, along these studies many useful tools were developed, such as the estimates on character sums $[2,7]$ and the large sieve inequalities $[18,21]$. Recently much attention is drawn to $G L_{2}$ analogues, and the generalizations include the first negative Hecke eigenvalues $[11,9,13,20]$, the recognition of newforms by values or signs of Hecke eigenvalues $[5,14,17,13,20]$, etc.

Let $k \geqslant 2$ be an even integer and $N \geqslant 1$ be an integer. We denote by $\mathrm{H}_{k}^{*}(N)$ the set of all primitive cusp forms of weight $k$ and of level $N$. For each integer $n \geqslant 1$, let $\lambda_{f}(n)$ be the Hecke eigenvalue of $f \in \mathrm{H}_{k}^{*}(N)$ under the Hecke operator $T_{n}$. The eigenvalues $\lambda_{f}(n)$ 's are real and verify the Hecke relation:

$$
\lambda_{f}(m) \lambda_{f}(n)=\sum_{\substack{d \mid(m, n) \\(d, N)=1}} \lambda_{f}\left(\frac{m n}{d^{2}}\right)
$$

for all integers $m \geqslant 1$ and $n \geqslant 1$. Note that $\lambda_{f}(1)=1$. The problem of the first negative Hecke eigenvalues is to evaluate the size of the least integer $n_{f}$ among all $n$ satisfying

$$
\lambda_{f}(n)<0 \quad \text { and } \quad(n, N)=1,
$$

for instance, to give a good bound for $n_{f}$ in term of conductor $k^{2} N$ of $f \in \mathrm{H}_{k}^{*}(N)$. This question was firstly studied by Kohnen \& Sengupta [11], and subsequently Iwaniec, Kohnen \& Sengupta [9] introduced a new method to achieve the "subconvexity bound" $\dagger$

$$
n_{f} \ll\left(k^{2} N\right)^{29 / 60} .
$$

Date: June 30, 2013.

2000 Mathematics Subject Classification. 11F30.

Key words and phrases. Fourier coefficients of modular forms.

$\dagger$ The convexity bound means the exponent $1 / 2$ in place of $29 / 60$, which is an immediate consequence of the convexity bound for Hecke $L$-function on the critical line. 
But interestingly, this bound is obtained without using any subconvexity bound for Hecke $L$-functions on the critical line. Their method has been refined very recently by Kowalski et al. [13] and by Matomäki [20], and the exponent 29/60 is improved to $9 / 20$ and $2 / 5$ respectively. The refinement of the method of Iwaniec, Kohnen \& Sengupta in $[13,20]$ makes use of the following three ingredients:

- Deligne's result: there is a real number $\theta_{f}(p) \in[0, \pi]$ such that

$$
\lambda_{f}(p)=2 \cos \theta_{f}(p) ;
$$

- The Hecke relation for $\lambda_{f}\left(p^{\nu}\right)$ in the form of

$$
\lambda_{f}\left(p^{\nu}\right)=\frac{\sin \left((\nu+1) \theta_{f}(p)\right)}{\sin \theta_{f}(p)} \quad(p \nmid N, \nu \geqslant 1) ;
$$

- The respective results for the density of integers without large and small prime factors, and the density of squarefree friable integers coprime with $N$.

This problem is further extended to higher rank cases. In this direction, $\mathrm{Qu}$ [22] obtained a polynomial bound: Let $m \geqslant 2$ be an integer, and $\pi$ an irreducible unitary cuspidal representation for $G L_{m}\left(\mathbb{A}_{\mathbb{Q}}\right)$ with arithmetic conductor $N_{\pi}$ and analytic conductor $Q_{\pi}$. We write $L(s, \pi)$ for the attached automorphic $L$-function and let $\left\{\lambda_{\pi}(n)\right\}_{n \geqslant 1}$ be the sequence of coefficients in the Dirichlet series of $L(s, \pi)$ in the half-plane $\Re e s>1$. Assume that the sequence $\left\{\lambda_{\pi}(n)\right\}_{n \geqslant 1}$ is real, and let $n_{\pi}$ be the least integer $n$ such that $\lambda_{\pi}(n)<0$. $\ddagger$ Qu derived the result $[22$, Theorem $1.2]$ that for any $\varepsilon>0$,

$$
n_{\pi} \ll_{m, \varepsilon} Q_{\pi}^{m / 2+\varepsilon}
$$

where the implied constant depends only on $m$ and $\varepsilon$, with her very elegant inequality [22, Lemma 5.3]

$$
\left|\lambda_{\pi}(p)\right|+\cdots+\left|\lambda_{\pi}\left(p^{m}\right)\right| \geqslant 1 / m \quad\left(p \nmid N_{\pi}\right) .
$$

Very recently the exponent $m / 2$ in (1.5) has been improved to 1 by Liu, $\mathrm{Qu} \& \mathrm{Wu}$ [19]. These results cover generic cases, but are weaker than the convexity bound when $m \geqslant 2$. Breaking the convexity is doubtless of deeper interest but no such result for $G L_{m}, m \geqslant 3$, is available in the literature.

In this paper we establish a subconvexity bound for a special case of $G L_{3}$ - the symmetric square lift of $G L_{2}$ forms. To each $f \in \mathrm{H}_{k}^{*}(N)$ is associated a symmetric square $L$-function, defined as

$$
L\left(s, \operatorname{sym}^{2} f\right):=\prod_{p}\left(1-\frac{\lambda_{f}\left(p^{2}\right)}{p^{s}}+\frac{\psi_{N}(p) \lambda_{f}\left(p^{2}\right)}{p^{2 s}}-\frac{\psi_{N}(p)}{p^{3 s}}\right)^{-1}=: \sum_{n \geqslant 1} \frac{\lambda_{\mathrm{sym}^{2} f}(n)}{n^{s}}
$$

for $\Re e s>1$, where $\psi_{N}$ denotes the principal character mod $N$ (cf. [24]). Inherited from the construction, $\lambda_{\text {sym }^{2} f}(n)$ is real, multiplicative and satisfies

$$
\lambda_{\text {sym }^{2} f}(n)=\sum_{d^{2} m=n} \lambda_{f}\left(m^{2}\right) \quad \text { for } \quad(n, N)=1 .
$$

Let us write $n_{\mathrm{sym}^{2} f}$ for the least integer $n$ such that

$$
\lambda_{\text {sym }^{2} f}(n)<0 \quad \text { and } \quad(n, N)=1 .
$$

\footnotetext{
${ }^{\ddagger}$ Here there is a slight difference from (1.2): without the extra condition $\left(n, N_{\pi}\right)=1$.
} 
By the work of Gelbart \& Jacquet [6], there is an irreducible unitary cuspidal representation $\pi$ for $G L_{3}\left(\mathbb{A}_{\mathbb{Q}}\right)$ such that $L\left(s, \operatorname{sym}^{2} f\right)=L(s, \pi)$. Thus Qu's bound $(1.5)$ with the refinement of [19] reads as

$$
n_{\mathrm{sym}^{2} f} \ll_{\varepsilon}\left(k^{3} N^{2}\right)^{1+\varepsilon},
$$

where the implied constant depends on $\varepsilon$ only. Extending the method of $[9,13,20]$, we derive a quite good subconvexity bound for $n_{\mathrm{sym}^{2} f}$.

Theorem 1. Let $k \geqslant 2$ be an even integer and $N \geqslant 1$ be an integer. Then for all $f \in \mathrm{H}_{k}^{*}(N)$, we have

$$
n_{\text {sym }^{2} f} \ll\left(k^{3} N^{2}\right)^{40 / 113},
$$

where the implied constant is absolute.

It is worth to notice that the exponent $40 / 113$ is smaller than the $G L_{2}$-exponent $2 / 5$ of Matomäki [20]. The underlying reason seems due to the methodology and the asymmetric distribution of $\lambda_{\operatorname{sym}^{2} f}(p)$, for $-1 \leqslant \lambda_{\mathrm{sym}^{2} f}(p) \leqslant 3$ while $-2 \leqslant \lambda_{f}(p) \leqslant 2$, the sum of $\lambda_{\text {sym }^{2} f}(n)$ over squarefree friable $n$ will heuristically bias towards positive more rapidly under the assumption $\lambda_{\mathrm{sym}^{2} f}(p) \geqslant 0$ for small $p$ 's.

Plainly $n_{\mathrm{sym}^{2} f}=p^{\nu}$ is a prime power due to the multiplicativity of $\lambda_{\mathrm{sym}^{2} f}(n)$. However unlike the least quadratic non-residues, we do not know whether the first negative coefficient of symmetric square $L$-function is attained at a prime argument (i.e. $\nu=1$ ). Let us introduce $n_{f, 2}$ for the least prime number $p \nmid N$ such that $\lambda_{\text {sym }^{2} f}(p)<0$. Clearly $n_{\mathrm{sym}^{2} f} \leqslant n_{f, 2}$. Under the Grand Riemann Hypothesis for $L\left(s, \operatorname{sym}^{2} f\right)$, one can show $n_{f, 2} \ll(\log (k N))^{2}$ where the implied constant is absolute. In [13], Kowalski et al. obtained an almost-all result: Let $k \geqslant 2$ be an even integer and $N \geqslant 1$ be a squarefree integer. There is a positive absolute constant $c$ such that

$$
n_{f, 2} \ll \log (k N)
$$

for all but except $O\left(k N e^{-c \log (k N) / \log _{2}(k N)}\right)$ forms $f \in \mathrm{H}_{k}^{*}(N)$. Here the implied constants in the $\ll$ and $O$-symbols are absolute. These conditional and almost all bounds for $n_{f, 2}$ also hold for $n_{\mathrm{sym}^{2} f}$, since $n_{\mathrm{sym}^{2} f} \leqslant n_{f, 2}$.

We end this section with an outline of the method. Similarly to $[9,13,20]$, let $y$ be the greatest integer such that

$$
\lambda_{\operatorname{sym}^{2} f}(n) \geqslant 0 \text { for } n \leqslant y \text { and }(n, N)=1,
$$

and consider

$$
S_{\mathrm{sym}^{2} f}\left(y^{u}\right):=\sum_{n \leqslant y^{u}}^{b} \lambda_{\mathrm{sym}^{2} f}(n)
$$

where $\sum^{b}:=\sum_{(n, N)=1} \mu(n)^{2}$ and $\mu(n)$ is the Möbius function. We shall obtain an estimate for $y$ by comparing the upper and lower bounds for $S_{\operatorname{sym}^{2} f}\left(y^{u}\right)$. The former is rather easy, and for the latter, the principle of the methods in $[9,13,20]$ is still effective. Nonetheless we need to invoke new identities and new tools in our manipulation. More precisely, with (1.6) and (1.4), we can prove that

$$
\lambda_{\mathrm{sym}^{2} f}\left(p^{\nu}\right)=\frac{\sin \left((\nu+2) \theta_{f}(p)\right) \sin \left((\nu+1) \theta_{f}(p)\right)}{\sin \theta_{f}(p) \sin \left(2 \theta_{f}(p)\right)} \quad(p \nmid N, \nu \geqslant 1) .
$$


However using merely this identity and the positivity hypothesis (1.10), we cannot derive directly the required lower bound for $\lambda_{\mathrm{sym}^{2} f}(p)$. We must exclude those primes $p$ for which $\lambda_{\mathrm{sym}^{2} f}\left(p^{\nu}\right)=0$ where $1 \leqslant \nu \leqslant 4$. (See Lemma 3.1 below for details.) Such primes are few, because it is equivalent to enumerate $p$ with $\lambda_{f}(p)=\alpha$ for a given algebraic number $\alpha \neq 0$. In fact, it was observed in [12] the sparsity of $p$ where $\lambda_{f}(p)= \pm 1$. Lemma 2.4 below is a generalization to suit our purpose.

Another technicality is the mean value of a multiplicative function $g$ over friable integers coprime to $q$ :

$$
\sum_{\substack{n \leqslant y^{u},(n, q)=1 \\ P(n) \leqslant y}} g(n),
$$

where $P(n)$ denotes the greatest prime factor of the integer $n$ with the convention that $P(1)=1$. There seems no handy reference in the literature. To this end we prove Lemma 4.2 below, in which the ranges of $q, u$ and $y$ are however rather weak. Much more general and better results will be obtained if one combines the methods in $[28,8,29]$ (where the case of $q=1$ is treated) and in [4] (where $g(n) \equiv 1$ ). This problem deserves more attention because of its own interest and future applications.

Acknowledgements. Lau and Liu wish to thank l'Institut Elie Cartan Nancy (IECN) for the hospitality and support during the preparation of this article. Lau is supported by General Research Fund (HKU 702308P) from the Research Grants Council of Hong Kong, and Liu is supported by the NSFC grant 11031004. We would like to acknowledge E. Kowalski for his unpublished work [12], which constitutes Section 2 here.

\section{ExCluding CERtain BAd Behavior of HeCke eigenvalues}

In order to bound $S_{\mathrm{sym}^{2} f}\left(y^{u}\right)$ from below, we need a control on small $\lambda_{\operatorname{sym}^{2} f}(p)$ which reduces, via (1.12), to remove the "bad" primes $p$, all contained in the set:

$$
\mathscr{P}_{f}:=\bigcup_{1 \leqslant \nu \leqslant 4}\left\{p:\left|\lambda_{f}(p)\right|=2 \cos (\pi /(\nu+2))\right\} .
$$

A general result of Serre [23, Theorem 15] implies that

$$
\left|\mathscr{P}_{f}\right| \ll_{f, \delta} \frac{x}{(\log x)^{1+\delta}}
$$

for all $\delta<1 / 2$ and $x \geqslant 2$. This bound is non-trivial, but unfortunately not sufficient for our purpose. Instead the unpublished work [12] of Kowalski is fitting more, and we devote this section to its slight generalization. Firstly we invoke a result of Besicovitch, see the lemma of Chandrasekharan in [3, p.204].

Lemma 2.1. (Besicovitch) Let $a_{j} \in \mathbb{Z}$ for $j=1, \ldots, r$ and $q_{j}=a_{j} p_{j}$ where $p_{1}, \ldots, p_{r}$ are distinct primes. Suppose that $\left(a_{j}, p_{1} \cdots p_{r}\right)=1$. ${ }^{\S}$ Then

$$
\sqrt{q_{j}} \notin \mathbb{Q}_{j}:=\mathbb{Q}\left(\sqrt{q_{1}}, \ldots, \sqrt{q_{j-1}}, \sqrt{q_{j+1}}, \ldots, \sqrt{q_{r}}\right) .
$$

Below is a direct consequence.

\footnotetext{
${ }^{\S}$ Remark that $(0, m)=m$.
} 
Lemma 2.2. Let $\mathbb{K}$ be a finite extension field over $\mathbb{Q}$. Then there are constants $M_{\mathbb{K}}, N_{\mathbb{K}} \in \mathbb{N}$ such that for any rational prime $p \nmid M_{\mathbb{K}}$ and for any a $\in \mathbb{Z}$ with $\left(a, p N_{\mathbb{K}}\right)=1$, we have $\sqrt{a p} \notin \mathbb{K}$.

Proof. Let $p_{1}, \ldots, p_{d}$ be distinct rational primes, and $a_{1}, \ldots, a_{d}$ be integers satisfy $\left(a_{1} \cdots a_{d}, p_{1} \cdots p_{d}\right)=1$. Then by Lemma 2.1 , we see that

$$
\left[\mathbb{Q}\left(\sqrt{a_{1} p_{1}}, \ldots, \sqrt{a_{d} p_{d}}\right): \mathbb{Q}\right]=2^{d},
$$

and hence, there is an upper bound for the number $r$ for which

$$
\mathbb{Q}\left(\sqrt{a_{1} p_{1}}, \ldots, \sqrt{a_{r} p_{r}}\right) \subset \mathbb{K}
$$

where $p_{1}, \ldots, p_{r}$ are distinct rational primes and $\left(a_{1} \cdots a_{r}, p_{1} \cdots p_{r}\right)=1$. Take $r$ to be the maximal value and let $a_{i}, p_{i}(i=1, \ldots, r)$ be a maximal set. We define

$$
N_{\mathbb{K}}=\prod_{1 \leqslant i \leqslant r} p_{i} \quad \text { and } \quad M_{\mathbb{K}}=N_{\mathbb{K}} \prod_{1 \leqslant i \leqslant r} a_{i} .
$$

Now, any $p \nmid M_{\mathbb{K}}$ and any $\left(a, p N_{\mathbb{K}}\right)=1$ satisfy $\left(a_{1} \cdots a_{r} a, p_{1} \cdots p_{r} p\right)=1$ and thus $\sqrt{a p} \notin \mathbb{Q}\left(\sqrt{a_{1} p_{1}}, \ldots, \sqrt{a_{r} p_{r}}\right)$. If $\sqrt{a p} \in \mathbb{K}$, it would follow

$$
\mathbb{Q}\left(\sqrt{a_{1} p_{1}}, \ldots, \sqrt{a_{r} p_{r}}, \sqrt{a p}\right) \subset \mathbb{K} .
$$

This contradicts to the maximality of $r$.

Next we deduce the following lemma by the argument in the proof of Lemma 2.1 in $[3]$.

Lemma 2.3. Let $\mathbb{K}$ be a finite extension field over $\mathbb{Q}$, and $M_{\mathbb{K}}, N_{\mathbb{K}}$ be the numbers same as in Lemma 2.2. Given any distinct rational primes $p_{1}, \ldots, p_{\ell} \nmid M_{\mathbb{K}}$, we have

$$
\sqrt{n p_{j}} \notin \mathbb{K}_{j}:=\mathbb{K}\left(\sqrt{p_{1}}, \ldots, \sqrt{p_{j-1}}, \sqrt{p_{j+1}}, \ldots, \sqrt{p_{\ell}}\right)
$$

for any integer $\left(n, p_{1} \cdots p_{\ell} N_{\mathbb{K}}\right)=1$ and any $j=1, \ldots, \ell$.

Proof. When $\ell=1$, we have $\mathbb{K}_{1}=\mathbb{K}$. This reduces to the case in Lemma 2.2, so the statement holds. Assume the induction hypothesis for the case of $\ell$ distinct primes.

Consider distinct primes $p_{1}, \ldots, p_{\ell+1} \nmid M_{\mathbb{K}}$ and suppose

$$
\sqrt{n p_{\ell+1}} \in \mathbb{K}\left(\sqrt{p_{1}}, \ldots, \sqrt{p_{\ell}}\right)=\mathbb{K}\left(\sqrt{p_{1}}, \ldots, \sqrt{p_{\ell-1}}\right)\left(\sqrt{p_{\ell}}\right)
$$

where $\left(n, p_{1} \cdots p_{\ell+1} N_{\mathbb{K}}\right)=1$. It follows that $\sqrt{n p_{\ell+1}}=\alpha+\beta \sqrt{p_{\ell}}$ where $\alpha, \beta \in$ $\mathbb{K}\left(\sqrt{p_{1}}, \ldots, \sqrt{p_{\ell-1}}\right)$, and consequently,

$$
2 \alpha \beta \sqrt{p_{\ell}}=n p_{\ell+1}-\alpha^{2}-\beta^{2} p_{\ell} \in \mathbb{K}\left(\sqrt{p_{1}}, \ldots, \sqrt{p_{\ell-1}}\right) .
$$

By the induction assumption, we infer that $\alpha=0$ or $\beta=0$, for otherwise we have $\sqrt{p_{\ell}} \in \mathbb{K}\left(\sqrt{p_{1}}, \ldots, \sqrt{p_{\ell-1}}\right)$.

If $\alpha=0$, then $\sqrt{n p_{\ell+1} p_{\ell}}=\beta p_{\ell} \in \mathbb{K}\left(\sqrt{p_{1}}, \ldots, \sqrt{p_{\ell-1}}\right)$. As $\left(n p_{\ell+1}, p_{1} \cdots p_{\ell} N_{\mathbb{K}}\right)=1$, it contradicts to the induction assumption. So $\beta=0$, and then we have that $\sqrt{n p_{\ell+1}} \in \mathbb{K}\left(\sqrt{p_{1}}, \ldots, \sqrt{p_{\ell-1}}\right)$. But now we apply the induction assumption to the $\ell$ distinct primes $p_{1}, \ldots, p_{\ell-1}, p_{\ell+1}$, we can infer that $\sqrt{n p_{\ell+1}} \notin \mathbb{K}\left(\sqrt{p_{1}}, \ldots, \sqrt{p_{\ell-1}}\right)$ since $\left(n, p_{1} \cdots p_{\ell-1} p_{\ell+1} N_{\mathbb{K}}\right)=1$. Contradiction arises again. Our proof is hence complete.

We come to the main result of this section - Lemma 2.4 - which is substantially verbatim from Kowalski [12], in view of his excellent elucidation. 
Lemma 2.4. Let $k \geqslant 2$ be an even integer and $N \geqslant 1$ be an integer. There is an absolute constant $C$ such that the inequality

$$
\left|\mathscr{P}_{f}\right| \leqslant \frac{4}{\log 2} \log (k N)+C
$$

holds for all $f \in \mathrm{H}_{k}^{*}(N)$.

Proof. We will need two basic facts on Fourier coefficients of primitive forms, which are essentially due to Shimura [25]:

- The field

$$
\mathbb{Q}_{f}=\mathbb{Q}\left(a_{f}(n)\right)_{n \geqslant 1}
$$

is a number field, where $a_{f}(n):=\lambda_{f}(n) n^{(k-1) / 2}$.

- For any automorphism $\sigma$ in the Galois group of $\overline{\mathbb{Q}}$ over $\mathbb{Q}$, the function

$$
f^{\sigma}:=\sum_{n \geqslant 1} \sigma\left(a_{f}(n)\right) \mathrm{e}^{2 \pi \mathrm{i} n z} \quad(\Im m z>0)
$$

is also an element of $\mathrm{H}_{k}^{*}(N)$. ¿From these two properties, we deduce first that

$$
\left[\mathbb{Q}_{f}: \mathbb{Q}\right] \leqslant\left|\mathrm{H}_{k}^{*}(N)\right|
$$

Indeed, notice that we have $f^{\sigma}=f$ if and only if $\sigma$ is in the subgroup of the Galois group of $\mathbb{Q}$ fixing $\mathbb{Q}_{f}$, so that the number of distinct conjugates $f$ is at most the index of this subgroup, or in other words the degree of the extension field $\mathbb{Q}_{f}$, while on the other hand there can be no more that $\left|\mathrm{H}_{k}^{*}(N)\right|$ distinct conjugates by the second property.

Now since the Fourier coefficients are real numbers, we have

$$
\left|\lambda_{f}(p)\right|=2 \cos (\pi /(\nu+2)) \Leftrightarrow a_{f}(p)= \pm 2 \cos (\pi /(\nu+2)) p^{(k-1) / 2} .
$$

Since $k$ is even, this implies in either case that $\cos (\pi /(\nu+2)) \sqrt{p} \in \mathbb{Q}_{f}$. Fix $1 \leqslant \nu \leqslant 4$ and write

$$
\alpha_{\nu}=\cos (\pi /(\nu+2)) \neq 0 .
$$

Set $\mathbb{K}=\mathbb{Q}\left(\alpha_{\nu}\right)$ and write $M_{\nu}=M_{\mathbb{K}}$ as defined in Lemma 2.3. Let $p_{1}<p_{2}<\cdots<p_{d}$ be distinct primes such that $p_{i} \nmid M_{\nu}$ and $\left|\lambda_{f}\left(p_{i}\right)\right|=2 \alpha_{\nu}$. It follows that

$$
\mathbb{Q}\left(\alpha_{\nu} \sqrt{p_{1}}, \ldots, \alpha_{\nu} \sqrt{p_{d}}\right) \subset \mathbb{Q}_{f} .
$$

Next we claim that

$$
\left[\mathbb{Q}\left(\alpha_{\nu} \sqrt{p_{1}}, \ldots, \alpha_{\nu} \sqrt{p_{d}}\right): \mathbb{Q}\right] \geqslant 2^{d}
$$

which is clearly true once

$$
\alpha_{\nu \sqrt{p_{j}}} \notin \mathbb{Q}\left(\alpha_{\nu \sqrt{p_{1}}}, \ldots, \alpha_{\nu} \sqrt{p_{j-1}}\right) \quad(j=1, \ldots, d) .
$$

Plainly,

$$
\mathbb{Q}\left(\alpha_{\nu} \sqrt{p_{1}}, \ldots, \alpha_{\nu} \sqrt{p_{j-1}}\right) \subset \mathbb{K}\left(\sqrt{p_{1}}, \ldots, \sqrt{p_{j-1}}\right),
$$

but by Lemma $2.3, \sqrt{p_{j}} \notin \mathbb{K}\left(\sqrt{p_{1}}, \ldots, \sqrt{p_{j-1}}\right)$ and neither does $\alpha_{\nu} \sqrt{p_{j}}$.

It follows from (2.4) and (2.5) that

$$
2^{d} \leqslant\left[\mathbb{Q}_{f}: \mathbb{Q}\right] \leqslant\left|\mathrm{H}_{k}^{*}(N)\right| \ll k N .
$$

with an absolute implied constant, we obtain the bound

$$
d \leqslant \frac{1}{\log 2} \log (k N)+O(1)
$$


Since there are at most $O\left(\log M_{\nu}\right)$ prime factors of $M_{\nu}$, the desired bound for $\left|\mathscr{P}_{f}\right|$ follows.

Remark. Bruinier \& Kohnen [1, Remark 2.3] gave a non-explicit form of (2.3) for the simpler case $\left|\lambda_{f}(p)\right|=2$. Some interesting applications of (2.3) and (2.2) are given in $[10,15]$.

\section{Proof of Theorem 1}

In this section, we prove Theorem 1 by assuming Lemma 3.2 below, whose proof will be given in Sections 4 and 5 .

We begin with the lower bounds for $\lambda_{\operatorname{sym}^{2} f}(p)$ under the positivity hypothesis (1.10). Define

$$
N_{f}:=\prod_{p \mid N} p \times \prod_{p \in \mathscr{P}_{f}} p
$$

where $\mathscr{P}_{f}$ is defined as in (2.1). Note that Lemma 2.4 implies

$$
\omega\left(N_{f}\right) \ll \log (k N)
$$

for all $f \in \mathrm{H}_{k}^{*}(N)$, where the implied constant is absolute. The symbol $\omega(n)$ denotes the number of distinct prime factors of $n$ with the convention $\omega(1)=0$.

Lemma 3.1. Let $k \geqslant 2$ be even integer and $N \geqslant 1$ be a positive integer.

(i) Formula (1.12) holds for all $f \in \mathrm{H}_{k}^{*}(N)$.

(ii) Let $y$ be defined as in (1.10) and $1 \leqslant \nu \leqslant 4$. Then for $p \leqslant y^{1 / \nu}$ and $p \nmid N_{f}$, we have

$$
\lambda_{\mathrm{sym}^{2} f}(p) \geqslant \kappa_{\nu}:=3-4 \sin ^{2}(\pi /(\nu+2)) .
$$

More precisely

$$
\lambda_{\text {sym }^{2} f}(p) \geqslant \begin{cases}0 & \text { if } y^{1 / 2}<p \leqslant y \text { and } p \nmid N_{f}, \\ 1 & \text { if } y^{1 / 3}<p \leqslant y^{1 / 2} \text { and } p \nmid N_{f}, \\ (\sqrt{5}+1) / 2 & \text { if } y^{1 / 4}<p \leqslant y^{1 / 3} \text { and } p \nmid N_{f}, \\ 2 & \text { if } p \leqslant y^{1 / 4} \text { and } p \nmid N_{f} .\end{cases}
$$

Proof. For $p \nmid N$ and $\nu \geqslant 1$, from (1.6) and (1.4) we can deduce that

$$
\lambda_{\mathrm{sym}^{2} f}\left(p^{2 \nu-1}\right)=\sum_{\ell=1}^{\nu} \lambda_{f}\left(p^{4 \ell-2}\right)=\sum_{\ell=1}^{\nu} \frac{\sin \left((4 \ell-1) \theta_{f}(p)\right)}{\sin \theta_{f}(p)} .
$$

By using the identity $2 \sin x \sin y=\cos (x-y)-\cos (x+y)$, it follows that

$$
\begin{aligned}
\lambda_{\mathrm{sym}^{2} f}\left(p^{2 \nu-1}\right) & =\sum_{\ell=1}^{\nu} \frac{\cos \left((4 \ell-3) \theta_{f}(p)\right)-\cos \left((4 \ell+1) \theta_{f}(p)\right)}{2 \sin \theta_{f}(p) \sin \left(2 \theta_{f}(p)\right)} \\
& =\frac{\cos \theta_{f}(p)-\cos \left((4 \nu+1) \theta_{f}(p)\right)}{2 \sin \theta_{f}(p) \sin \left(2 \theta_{f}(p)\right)} .
\end{aligned}
$$

Using the preceding identity again yields

$$
\lambda_{\mathrm{sym}^{2} f}\left(p^{2 \nu-1}\right)=\frac{\sin \left((2 \nu+1) \theta_{f}(p)\right) \sin \left(2 \nu \theta_{f}(p)\right)}{\sin \theta_{f}(p) \sin \left(2 \theta_{f}(p)\right)} .
$$


This proves Part (i), as a similar argument applies to

$$
\lambda_{\mathrm{sym}^{2} f}\left(p^{2 \nu}\right)=\frac{\sin \left((2 \nu+2) \theta_{f}(p)\right) \sin \left((2 \nu+1) \theta_{f}(p)\right)}{\sin \theta_{f}(p) \sin \left(2 \theta_{f}(p)\right)}
$$

for $p \nmid N$ and $\nu \geqslant 1$.

Now we know

$$
\lambda_{\mathrm{sym}^{2} f}(p)=\frac{\sin \left(3 \theta_{f}(p)\right)}{\sin \theta_{f}(p)}=3-4 \sin ^{2} \theta_{f}(p) .
$$

In view of the definitions of $y$ and $N_{f}$, we have that for $1 \leqslant \nu \leqslant 4$ and $p \leqslant y^{1 / \nu}$ with $p \nmid N_{f}$,

$$
\frac{\lambda_{\mathrm{sym}^{2} f}\left(p^{j}\right)}{\lambda_{\mathrm{sym}^{2} f}\left(p^{j-1}\right)}=\frac{\sin \left((j+2) \theta_{f}(p)\right)}{\sin \left(j \theta_{f}(p)\right)}>0 \quad(1 \leqslant j \leqslant \nu),
$$

recalling $\lambda_{\text {sym }^{2} f}(1)=1$. The case $j=1$ implies

$$
0 \leqslant \theta_{f}(p)<\pi / 3 \quad \text { or } \quad 2 \pi / 3<\theta_{f}(p) \leqslant \pi
$$

$\left(\operatorname{as} \theta_{f}(p) \in[0, \pi]\right)$. Observe that

$$
\frac{\sin \left((2 \ell+1) \theta_{f}(p)\right)}{\sin \theta_{f}(p)}=\prod_{j=1}^{\ell} \frac{\lambda_{\mathrm{sym}^{2} f}\left(p^{2 j-1}\right)}{\lambda_{\mathrm{sym}^{2} f}\left(p^{2 j-2}\right)}
$$

and

$$
\frac{\sin \left((2 \ell+2) \theta_{f}(p)\right)}{\sin \left(2 \theta_{f}(p)\right)}=\prod_{j=1}^{\ell} \frac{\lambda_{\mathrm{sym}^{2} f}\left(p^{2 j}\right)}{\lambda_{\mathrm{sym}^{2} f}\left(p^{2 j-1}\right)} .
$$

If $0 \leqslant \theta_{f}(p)<\pi / 3$, then both $\sin \theta_{f}(p)$ and $\sin \left(2 \theta_{f}(p)\right)>0$. A successive application of the positivity with the last two formulas yields $\sin \left((\ell+2) \theta_{f}(p)\right)>0$ for all $1 \leqslant \ell \leqslant \nu$, and hence $0 \leqslant \theta_{f}(p)<\pi /(\nu+2)$. In case $2 \pi / 3<\theta_{f}(p) \leqslant \pi$, we take $\vartheta_{f}(p)=\pi-\theta_{f}(p) \in[0, \pi / 3)$, then the above formulas hold for $\vartheta_{f}(p)$ in place of $\theta_{f}(p)$, with $\sin \vartheta_{f}(p)>0$ and $\sin \left(2 \vartheta_{f}(p)\right)>0$. We hence obtain that $(\nu+1) \pi /(\nu+2)<$ $\theta_{f}(p) \leqslant \pi$.

Thus (3.3) follows with (3.5), and (3.4) is also an immediate consequence, for $\kappa_{1}=0, \kappa_{2}=1, \kappa_{3}=(\sqrt{5}+1) / 2$ and $\kappa_{4}=2$.

In view of (3.4) and the fact

$$
\lambda_{\text {sym }^{2} f}(p)=\lambda_{f}\left(p^{2}\right)=\lambda_{f}(p)^{2}-1 \geqslant-1 \quad(p \nmid N),
$$

we introduce the auxiliary multiplicative function $h=h_{N_{f}, y}$ defined as

$$
h_{N_{f}, y}(p)= \begin{cases}-1 & \text { if } p>y \text { and } p \nmid N_{f}, \\ 0 & \text { if } y^{1 / 2}<p \leqslant y \text { or } p \mid N_{f}, \\ 1 & \text { if } y^{1 / 3}<p \leqslant y^{1 / 2} \text { and } p \nmid N_{f}, \\ (\sqrt{5}+1) / 2 & \text { if } y^{1 / 4}<p \leqslant y^{1 / 3} \text { and } p \nmid N_{f}, \\ 2 & \text { if } p \leqslant y^{1 / 4} \text { and } p \nmid N_{f},\end{cases}
$$

and $h_{N_{f}, y}\left(p^{\nu}\right)=0$ for all $p$ and $\nu \geqslant 2$. The key to obtain the required lower bound for $S_{\mathrm{sym}^{2} f}\left(y^{u}\right)$ is to evaluate the mean value of $h_{N_{f}, y}(n)$ (as shown in (3.11) below). The size of this mean value is related to the solution of a difference-differential equation. 
Let $\kappa \geqslant 1$ and $\rho_{\kappa}(t)$ be the unique continuous solution of the difference-differential equation

$$
\begin{cases}\rho_{\kappa}(t)=t^{\kappa-1} / \Gamma(\kappa) & (0 \leqslant t \leqslant 1) \\ \left(t^{1-\kappa} \rho_{\kappa}(t)\right)^{\prime}=-\kappa t^{-\kappa} \rho_{\kappa}(t-1) & (t>1)\end{cases}
$$

where $\Gamma(\kappa)$ denotes the gamma function. By [26, Lemma 4.2], if $\kappa \geqslant 1, \rho_{\kappa}(t)$ is increasing on $\left[0, t_{\kappa}\right]$ and decreasing on $\left[t_{\kappa}, \infty\right)$ where $\max \{1, \kappa-1\} \leqslant t_{\kappa} \leqslant \kappa$; furthermore, $\rho_{\kappa}(t) \rightarrow 0$ as $t \rightarrow \infty$. In particular we note that $t_{2}=\sqrt{\mathrm{e}}$.

Define

$$
\Pi_{q, \kappa}:=\left(\frac{\varphi(q)}{q}\right)^{\kappa} \prod_{p \nmid q}\left(1-\frac{1}{p}\right)^{\kappa}\left(1+\frac{\kappa}{p}\right),
$$

where $\varphi(n)$ is the Euler totient function. We have the following lemma, proven in Section 5 .

Lemma 3.2. With the previous notation, we have

$$
\sum_{n \leqslant y^{u}} h_{N_{f}, y}(n) \geqslant \Pi_{N_{f}, 2} y^{u}\left(\log y^{1 / 4}\right) \delta(u)\left\{1+O\left(\frac{\left(\log _{2} y\right)^{5}}{\log y}\right)\right\}
$$

uniformly for

$$
\frac{4}{3} \leqslant u \leqslant \frac{3}{2} \quad \text { and } \quad y \geqslant\left(k^{3} N^{2}\right)^{1 / 100}
$$

where

$$
\delta(u):=\delta_{1}(u)+\delta_{2}(u)+\delta_{3}(u)-\delta_{4}(u)
$$

and

$$
\begin{aligned}
\delta_{1}(u):= & \rho_{2}(4 u), \\
\delta_{2}(u):= & \kappa_{3} \int_{1}^{4 / 3} \frac{\rho_{2}(4 u-t)}{t} \mathrm{~d} t+\int_{4 / 3}^{2} \frac{\rho_{2}(4 u-t)}{t} \mathrm{~d} t, \\
\delta_{3}(u):= & \kappa_{3}^{2} \int_{1}^{4 / 3} \frac{\mathrm{d} t}{t} \int_{t}^{4 / 3} \frac{\rho_{2}(4 u-t-s)}{s} \mathrm{~d} s \\
& +\kappa_{3} \int_{1}^{4 / 3} \frac{\mathrm{d} t}{t} \int_{4 / 3}^{2} \frac{\rho_{2}(4 u-t-s)}{s} \mathrm{~d} s+\int_{4 / 3}^{2} \frac{\mathrm{d} t}{t} \int_{t}^{2} \frac{\rho_{2}(4 u-t-s)}{s} \mathrm{~d} s, \\
\delta_{4}(u):= & \int_{4}^{4 u} \frac{\rho_{2}(4 u-t)}{t} \mathrm{~d} t+\kappa_{3} \int_{4}^{4 u-4 / 3} \frac{\mathrm{d} t}{t} \int_{1}^{4 / 3} \frac{4 u-t-s}{s} \mathrm{~d} s \\
& +\int_{4}^{4 u-4 / 3} \frac{\mathrm{d} t}{t} \int_{4 / 3}^{4 u-t} \frac{4 u-t-s}{s} \mathrm{~d} s+\kappa_{3} \int_{4 u-4 / 3}^{4 u-1} \frac{\mathrm{d} t}{t} \int_{1}^{4 u-t} \frac{4 u-t-s}{s} \mathrm{~d} s
\end{aligned}
$$

with $\kappa_{3}=(\sqrt{5}+1) / 2$. The function $\delta(u)$ is decreasing on $[1+\sqrt{\mathrm{e}} / 4,3 / 2]$ and $\delta(u)>0$ for all $u<u_{0}$, where $u_{0}$ is the solution to $\delta\left(u_{0}\right)=0$ in $[1+\sqrt{\mathrm{e}} / 4,3 / 2]$. We have $u_{0}>113 / 80$. 
Now we are ready to show Theorem 1 with the help of Lemma 3.2. Let us start with the lower bound for $S_{\mathrm{sym}^{2} f}\left(y^{u}\right)$. As in [13], we notice that

$$
S_{\mathrm{sym}^{2} f}\left(y^{u}\right) \geqslant \sum_{n \leqslant y^{u}} h_{N_{f}, y}(n)
$$

for all $u<u_{0}$, provided $y$ is large enough, for instance, $y \geqslant\left(k^{3} N^{2}\right)^{1 / 100}$ and $k N$ is large enough, which can obviously be assumed in proving Theorem 1.

To see (3.11), let $g_{N_{f}, y}$ be the multiplicative function defined by the Dirichlet convolution identity $\lambda_{\mathrm{sym}^{2} f}=g_{N_{f}, y} * h_{N_{f}, y}$. Then $g_{N_{f}, y}(n) \geqslant 0$ for all squarefree integers $n \geqslant 1$ with $\left(n, N_{f}\right)=1$, since $g_{N_{f}, y}(p)=\lambda_{\text {sym }^{2} f}(p)-h_{N_{f}, y}(p) \geqslant 0$ for $p \nmid N_{f}$. This is easily verified from the definition of $h_{N_{f}, y}$, (3.4) and (3.6).

According to Lemma 3.2, we have

$$
\sum_{n \leqslant y^{u}} h_{N_{f}, y}(n) \geqslant 0
$$

for $u \leqslant u_{0}$ and sufficiently large $y$. But, as $g_{N_{f}, y}(1)=1$, we infer that

$$
\begin{aligned}
S_{\mathrm{sym}^{2} f}\left(y^{u}\right) & =\sum_{n \leqslant y^{u}}^{b} g_{N_{f}, y} * h_{N_{f}, y}(n) \\
& =\sum_{d \leqslant y^{u}}^{b} g_{N_{f}, y}(d) \sum_{m \leqslant y^{u} / d}^{b} h_{N_{f}, y}(m) \\
& \geqslant \sum_{m \leqslant y^{u}}^{b} h_{N_{f}, y}(m),
\end{aligned}
$$

which is (3.11). Then we deduce from Lemma 3.2 the required lower bound

$$
S_{\mathrm{sym}^{2} f}\left(y^{u}\right) \gg \frac{y^{u} \log y}{\left\{\log _{2}(k N)\right\}^{2}} \quad\left(u<u_{0}\right),
$$

since we have, by (3.8) and (3.2),

$$
\Pi_{N_{f}, 2} \gg\left\{\log \left(\omega\left(N_{f}\right)+3\right)\right\}^{-2} \gg\left\{\log _{2}(k N)\right\}^{-2} .
$$

Next we establish an upper bound for $S_{\mathrm{sym}^{2} f}\left(y^{u}\right)$. For $\Re e s>1$, we have

$$
\sum_{n \geqslant 1}^{b} \frac{\lambda_{\mathrm{sym}^{2} f}(n)}{n^{s}}=\prod_{p \nmid N}\left(1+\frac{\lambda_{f}\left(p^{2}\right)}{p^{s}}\right)=L\left(s, \operatorname{sym}^{2} f\right) G_{f}(s),
$$

where the Dirichlet series of

$$
G_{f}(s):=\prod_{p \mid N}\left(1-\frac{\lambda_{f}\left(p^{2}\right)}{p^{s}}\right) \prod_{p \nmid N}\left(1-\frac{\lambda_{f}\left(p^{2}\right)^{2}-\lambda_{f}\left(p^{2}\right)}{p^{2 s}}+\frac{\lambda_{f}\left(p^{2}\right)^{2}-1}{p^{3 s}}-\frac{\lambda_{f}\left(p^{2}\right)}{p^{4 s}}\right)
$$

converges absolutely and so $G_{f}(s) \ll_{\varepsilon} N^{\varepsilon}$ in the half-plane $\Re e s \geqslant 1 / 2+\varepsilon$ and $G_{f}(s) \ll_{\varepsilon} 1$ for $\Re e s \geqslant 1+\varepsilon\left(\right.$ as $\left|\lambda_{f}\left(p^{\nu}\right)\right| \leqslant \nu+1$ by Deligne's inequality).

The Perron formula (cf. [27, Theorem II.2.3]) gives

$$
\sum_{n \leqslant x}^{b} \lambda_{\mathrm{sym}^{2} f}(n)=\frac{1}{2 \pi \mathrm{i}} \int_{\kappa-\mathrm{i} T}^{\kappa+\mathrm{i} T} L\left(s, \operatorname{sym}^{2} f\right) G_{f}(s) \frac{x^{s}}{s} \mathrm{~d} s+O\left(x^{\varepsilon}\left(1+\frac{x}{T}\right)\right)
$$


where $\kappa=1+\varepsilon$. Using the convexity bound

$$
L\left(s, \operatorname{sym}^{2} f\right) \ll_{\varepsilon}\left(k^{3} N^{2}\left(|\tau|^{3}+1\right)\right)^{1 / 4+\varepsilon} \quad\left(s=\frac{1}{2}+\varepsilon+\mathrm{i} \tau, \tau \in \mathbb{R}\right),
$$

we move the line of integration $\Re e s=\kappa$ to $\Re e s=\frac{1}{2}+\varepsilon$ and select $T=x^{\varepsilon}$ to deduce that for $x \leqslant\left(k^{3} N^{2}\right)^{1 / 2+\varepsilon}$,

$$
S_{\mathrm{sym}^{2} f}(x) \ll_{\varepsilon}\left(k^{3} N^{2}\right)^{1 / 4+\varepsilon} x^{1 / 2+\varepsilon} .
$$

Now, a comparison with (3.12) gives the estimate

$$
y \leqslant\left(k^{3} N^{2}\right)^{1 /\left(2 u_{0}\right)+\varepsilon} .
$$

Quoting the lower bound for $u_{0}$ from Lemma 3.2, the proof of Theorem 1 is done.

\section{Mean value of multiplicative Function over fRiable integers COPRIME WITH $q$}

We prepare for the proof of Lemma 3.2. To this end we consider a mean value theorem of the multiplicative function $n \mapsto \mu(n)^{2} \kappa^{\omega(n)}$ over friable integers coprime with $q$, where $\kappa>0$ is a constant. For $x \geqslant 1, y \geqslant 2$ and $q \in \mathbb{N}$, define

$$
\Xi_{q, \kappa}(x, y):=\sum_{\substack{n \leqslant x,(n, q)=1 \\ P(n) \leqslant y}} \mu(n)^{2} \kappa^{\omega(n)} \quad \text { and } \quad \Xi_{q, \kappa}(x):=\Xi_{q, \kappa}(x, x) .
$$

We begin with the treatment of $\Xi_{q, \kappa}(x)$.

Lemma 4.1. Under the previous notation, there is a positive constant $C=C(\kappa)$ depending only on $\kappa$ such that we have

$$
\Xi_{q, \kappa}(x)=\frac{\Pi_{q, \kappa}}{\Gamma(\kappa)} x(\log x)^{\kappa-1}\left\{1+O_{\kappa}\left(\frac{L_{q}^{\mathrm{e} \kappa+2}}{\log x}\right)\right\}
$$

uniformly for

$$
q \geqslant 1 \quad \text { and } \quad x \geqslant \exp \left(C L_{q}^{\mathrm{e} \kappa+2}\right),
$$

where $\Pi_{q, \kappa}$ is defined as in (3.8) and

$$
L_{q}:=\log (\omega(q)+3) .
$$

Proof. For $\Re$ e $s>1$, we have

$$
\sum_{\substack{n=1 \\(n, q)=1}}^{\infty} \mu(n)^{2} \kappa^{\omega(n)} n^{-s}=\prod_{p \nmid q}\left(1+\kappa p^{-s}\right)=\zeta(s)^{\kappa} G_{q}(s),
$$

where $\zeta(s)$ is the Riemann $\zeta$-function and

$$
G_{q}(s):=\prod_{p \mid q}\left(1-p^{-s}\right)^{\kappa} \prod_{p \nmid q}\left(1-p^{-s}\right)^{\kappa}\left(1+\kappa p^{-s}\right)
$$

converges absolutely for $\Re e s \geqslant 1 / 2+\varepsilon$ and any $\varepsilon>0$.

\footnotetext{
${ }^{\ddagger}$ In [13], the inequality sign of " $x \geqslant Q^{2 \eta+\varepsilon " ~ b e l o w ~(2.1) ~ s h o u l d ~ b e ~ r e v e r s e d, ~ a n d ~} Q$ tacitly means $k^{2} N\left(1+|t|^{2}\right)$.
} 
By the Perron formula (see [27, Theorem II.2.3]), we can write

$$
\Xi_{q, \kappa}(x)=\frac{1}{2 \pi \mathrm{i}} \int_{b-\mathrm{i} T}^{b+\mathrm{i} T} \zeta(s)^{2} G_{q}(s) \frac{x^{s}}{s} \mathrm{~d} s+O\left(\mathcal{R}_{1}\right),
$$

where $b=1+1 / \log x, T \geqslant 3$ and

$$
\mathcal{R}_{1}:=x \sum_{n \geqslant 1} \frac{\kappa^{\omega(n)}}{n^{b}(1+T|\log (x / n)|)} .
$$

The implied constant in the $O$-term is absolute.

The summation of $\mathcal{R}_{1}$ over $n$ with $|\log (x / n)| \leqslant T^{-1 / 2}$ is

$$
\begin{aligned}
& \ll_{\kappa} \sum_{|n-x| \leqslant x T^{-1 / 2}} \kappa^{\omega(n)} \\
& \ll_{\kappa}\left(\sum_{|n-x| \leqslant x T^{-1 / 2}} \kappa^{2 \omega(n)}\right)^{1 / 2}\left(\sum_{|n-x| \leqslant x T^{-1 / 2}} 1\right)^{1 / 2} \\
& \ll_{\kappa} \frac{x(\log x)^{\left(\kappa^{2}-1\right) / 2}}{T^{1 / 4}},
\end{aligned}
$$

and the remaining part of $\mathcal{R}_{1}$, contributed from the sum over $n$ with $|\log (x / n)|>$ $T^{-1 / 2}$, is

$$
\ll_{\kappa} \frac{x}{T^{1 / 2}} \sum_{|n-x|>x T^{-1 / 2}} \frac{\kappa^{\omega(n)}}{n^{b}} \ll_{\kappa} \frac{x(\log x)^{\kappa-1}}{T^{1 / 2}} .
$$

As a result, we have

$$
\mathcal{R}_{1} \ll_{\kappa} \frac{x(\log x)^{c_{1}(\kappa)}}{T^{1 / 4}},
$$

where and in the sequel, $c_{i}(\kappa)(i=1,2, \ldots)$ denotes a positive constant depending only on $\kappa$.

It remains to evaluate the integral on the right-hand side of (4.3). Let $c$ be a suitable positive constant and

$$
\sigma(T):=1-c / \log T \text {. }
$$

Let $r=1 /(2 \log x)$ and assume $1-r>\sigma(T)$. The truncated Hankel contour $\Gamma$ is a positively oriented contour formed from the circle $|s-1|=r$ excluding the point $s=1-r$ and joining with the half-segment $[\sigma(T), 1-r]$ which is traced out twice with respective arguments $+\pi$ and $-\pi$. We apply the residue theorem to the integral over the closed path that consists of the vertical line segments $[b-\mathrm{i} T, b+\mathrm{i} T]$ and $\mathcal{L}_{\mathrm{v}}^{ \pm}:=[\sigma(T), \sigma(T) \pm \mathrm{i} T]$, two horizontal line segments $\mathcal{L}_{\mathrm{h}}^{ \pm}:=[\sigma(T)+\mathrm{i} T, b \pm \mathrm{i} T]$ and the contour $\Gamma$.

For $\Re e s \geqslant \sigma(T)$, we have

$$
\begin{aligned}
\left|G_{q}(s)\right| & \leqslant \prod_{p \mid q}\left(1+p^{-\sigma(T)}\right)^{\kappa} \\
& \leqslant \exp \left\{\kappa \sum_{p \leqslant p_{\omega(q)}} p^{-\sigma(T)}\right\} \\
& \ll \exp \left\{\kappa p_{\omega(q)}^{c / \log T} \log _{2} p_{\omega(q)}\right\},
\end{aligned}
$$


where $p_{n}$ is the $n$th prime. Since $p_{n} \sim n \log n$ by prime number theorem, we have

$$
\left|G_{q}(s)\right| \ll \exp \left\{\kappa \exp \left(2 c L_{q} / \log T\right) \log L_{q}\right\} \ll L_{q}^{\mathrm{e} \kappa}
$$

provided

$$
T \geqslant \exp \left\{2 c L_{q}\right\}
$$

Together with the well-known bound $\zeta(s) \ll \log T$ for $s \in \mathcal{L}_{\mathrm{h}}^{ \pm} \cup \mathcal{L}_{\mathrm{v}}^{ \pm} \cup \Gamma$, it follows that

$$
\int_{\mathcal{L}_{\mathrm{h}}^{ \pm} \cup \mathcal{L}_{\mathrm{v}}^{ \pm}} \zeta(s)^{\kappa} G_{q}(s) \frac{x^{s}}{s} \mathrm{~d} s \ll L_{q}^{\mathrm{e} \kappa}\left(\frac{x}{T}+x^{\sigma(T)}\right)(\log T)^{\kappa+1}
$$

if (4.6) is satisfied.

By (4.5) and the properties of $\zeta(s)$, we have

$$
s^{-1}((s-1) \zeta(s))^{\kappa} G_{q}(s)=G_{q}(1)+O_{\kappa}\left(L_{q}^{\mathrm{e} \kappa}|s-1|\right)
$$

for $s \in \Gamma$, under the hypothesis (4.6). The error term contributes a term

$$
\begin{aligned}
& \ll L_{q}^{\mathrm{e} \kappa} \int_{\Gamma}\left|(s-1)^{1-\kappa} x^{s}\right||\mathrm{d} s| \\
& \ll L_{q}^{\mathrm{e} \kappa} \int_{\sigma(T)}^{1-r}(1-\sigma)^{1-\kappa} x^{\sigma} \mathrm{d} \sigma+x^{1+r} r^{2-\kappa} \\
& \ll_{\kappa} L_{q}^{\mathrm{e} \kappa} x(\log x)^{\kappa-2} .
\end{aligned}
$$

By [27, Corollary II.5.2.1], we get from $G_{q}(1)$ the main term,

$$
\frac{G_{q}(1)}{2 \pi \mathrm{i}} \int_{\Gamma}(s-1)^{-\kappa} x^{s} \mathrm{~d} s=\frac{G_{q}(1)}{\Gamma(\kappa)} x(\log x)^{\kappa-1}\left\{1+O_{\kappa}\left(\mathrm{e}^{-c(\log x) / \log T}\right)\right\} .
$$

Combining them gives

$$
\begin{aligned}
\frac{1}{2 \pi \mathrm{i}} \int_{\kappa-\mathrm{i} T}^{\kappa+\mathrm{i} T} \zeta(s)^{\kappa} G_{q}(s) \frac{x^{s}}{s} \mathrm{~d} s & =\frac{G_{q}(1)}{\Gamma(\kappa)} x(\log x)^{\kappa-1}\left\{1+O\left(\mathrm{e}^{-c(\log x) / \log T}\right)\right\} \\
& +O_{\kappa}\left(L_{q}^{\mathrm{e} \kappa}\left(\frac{x}{T}+x^{\sigma(T)}\right)(\log T)^{\kappa+1}+L_{q}^{\mathrm{e} \kappa} x(\log x)^{\kappa-2}\right)
\end{aligned}
$$

under the hypothesis (4.6).

Inserting into (4.3) with (4.4), we obtain that

$$
\Xi_{q, \kappa}(x)=\frac{G_{q}(1)}{\Gamma(\kappa)} x(\log x)^{\kappa-1}+O_{\kappa}\left(\mathcal{R}_{2}\right)
$$

where

$$
\mathcal{R}_{2}:=\frac{G_{q}(1) x(\log x)^{\kappa-1}}{\mathrm{e}^{c(\log x) / \log T}}+L_{q}^{\mathrm{e} \kappa}\left(\frac{x}{T^{1 / 4}}+x^{\sigma(T)}\right)(\log T)^{c_{2}(\kappa)}+L_{q}^{\mathrm{e} \kappa} x(\log x)^{\kappa-2}
$$

if (4.6) holds.

It is easy to see that

$$
\Pi_{q, \kappa}=G_{q}(1) \gg L_{q}^{-2}
$$

We take

$$
T=\exp \left\{c_{3}(\kappa)(\log x)^{1 / 2}\right\}
$$


then the condition (4.6) holds valid since $x \geqslant \exp \left(C L_{q}^{2}\right)$. Moreover we can easily see that

$$
\mathcal{R}_{2} \ll_{\kappa} L_{q}^{-2} x(\log x)^{\kappa-1} \frac{L_{q}^{\mathrm{e} \kappa+2}}{\log x} \ll_{\kappa} \Pi_{q, \kappa} x(\log x)^{\kappa-1} \frac{L_{q}^{\mathrm{e} \kappa+2}}{\log x}
$$

uniformly for $q$ and $x$ verifying (4.1). The required result follows from (4.8) into $(4.7)$.

The next lemma plays a key role in the proof of Lemma 3.2. As mentioned in the introduction, we do not make effort to widen the ranges of the parameters involved.

Lemma 4.2. Let $\kappa \geqslant 1$ and $U>1$ be two fixed constants. For some suitable constant $C=C(\kappa, U)$ depending only on $\kappa$ and $U$, we have

$$
\Xi_{q, \kappa}\left(y^{u}, y\right)=\Pi_{q, \kappa} y^{u}(\log y)^{\kappa-1} \rho_{\kappa}(u)\left\{1+O_{\kappa, U}\left(\frac{L_{q}^{\mathrm{e} \kappa+2}\left(\log _{2} y\right)^{\delta_{\kappa, 1}}}{\log y}\right)\right\}
$$

uniformly for

$$
q \geqslant 1, \quad y \geqslant \exp \left(2 C L_{q}^{\mathrm{e} \kappa+2}\right), \quad U^{-1} \leqslant u \leqslant U
$$

where $\Pi_{q, \kappa}, L_{q}$ and $\rho_{\kappa}(u)$ are defined as in (3.8), (4.2) and (3.7), respectively, and

$$
\delta_{\kappa, 1}:= \begin{cases}1 & \text { if } \kappa=1 \\ 0 & \text { otherwise }\end{cases}
$$

Proof. If $U^{-1} \leqslant u \leqslant 1$, we have $\Xi_{q, \kappa}\left(y^{u}, y\right)=\Xi_{q, \kappa}\left(y^{u}\right)$. Thus Lemma 4.1 gives us immediately the required asymptotic formula since $\rho_{\kappa}(u)=u^{\kappa-1} / \Gamma(\kappa)$.

Next we suppose that $1 \leqslant u \leqslant 2$. Write

$$
\Xi_{q, \kappa}\left(y^{u}, y\right)=\Xi_{q, \kappa}\left(y^{u}\right)-\kappa \sum_{\substack{y<p \leqslant y^{u} \\ p \nmid q}} \Xi_{q, \kappa}\left(y^{u} / p\right) .
$$

With the help of Lemma 4.1, we have

$$
\Xi_{q, \kappa}\left(y^{u}\right)=\Pi_{q, \kappa} y^{u}(\log y)^{\kappa-1} \frac{u^{\kappa-1}}{\Gamma(\kappa)}\left\{1+O_{\kappa}\left(\frac{L_{q}^{\mathrm{e} \kappa+2}}{\log y}\right)\right\}
$$

and so

$$
\begin{aligned}
& \sum_{y<p \leqslant y^{u} \mathrm{e}^{-C L_{q}^{\mathrm{e} \kappa+2}}} \Xi_{q, \kappa}\left(y^{u} / p\right) \\
= & \sum_{y<p \leqslant y^{u} \mathrm{e}^{-C L_{q}^{\mathrm{e} \kappa+2}}} \Pi_{q, \kappa} \frac{y^{u}\left\{\log \left(y^{u} / p\right)\right\}^{\kappa-1}}{\Gamma(\kappa) p}\left\{1+O\left(\frac{L_{q}^{\mathrm{e} \kappa+2}}{\log \left(y^{u} / p\right)}\right)\right\} .
\end{aligned}
$$

The $O$-terms are absorbed in the $O$-term of (4.9) by partial integration with the prime number theorem and the fact that $\rho_{\kappa}(u) \gg_{\kappa} 1$ uniformly for $1 \leqslant u \leqslant 2$. The 
main term is

$$
\begin{gathered}
\sum_{y<p \leqslant y^{u} \mathrm{e}^{-C L_{q}^{\mathrm{e} \kappa+2}}} \Pi_{q, \kappa} \frac{y^{u}(\log p)^{\kappa-1}}{p} \rho_{\kappa}\left(\frac{\log \left(y^{u} / p\right)}{\log p}\right) \\
=\prod_{q, \kappa} y^{u}\left(\log y^{u}\right)^{\kappa-1} \int_{1}^{u} \frac{\rho_{\kappa}(t-1)}{t^{\kappa}} \mathrm{d} t\left\{1+O\left(\frac{L_{q}^{\mathrm{e} \kappa+2}}{\log y}\right)\right\} .
\end{gathered}
$$

On the other hand, we have

$$
\begin{aligned}
& \left(\sum_{\substack{y<p \leqslant y^{u} \\
p \mid q}}+\sum_{y^{u} \mathrm{e}^{-C L_{q}^{\mathrm{e} \kappa+2}}<p \leqslant y^{u}}\right) \Xi_{q, \kappa}\left(y^{u} / p\right) \\
& \ll y^{u}\left(\sum_{\substack{y<p \leqslant y^{u} \\
p \mid q}}+\sum_{y^{u} \mathrm{e}^{-C L_{q}^{\mathrm{e} \kappa+2}}<p \leqslant y^{u}}\right) \frac{\left\{\log \left(y^{u} / p\right)\right\}^{\kappa-1}}{p} \\
& \ll_{\kappa} y^{u}(\log y)^{\kappa-1}\left(\frac{L_{q}^{\mathrm{e} \kappa+2}}{\log y}\right)^{\kappa}
\end{aligned}
$$

which is admissible, for $\log y \gg L_{q}^{\mathrm{e} \kappa+2}$. Inserting these estimates into (4.11) and noticing that

$$
\rho_{\kappa}(u)=u^{\kappa-1}\left(\frac{1}{\Gamma(\kappa)}-\kappa \int_{1}^{u} \frac{\rho_{\kappa}(t-1)}{t^{\kappa}} \mathrm{d} t\right) \quad(1 \leqslant u \leqslant 2),
$$

we find that the asymptotic formula (4.9) holds uniformly for $q \geqslant 1, y \geqslant \exp \left(C L_{q}^{\mathrm{e} \kappa+2}\right)$ and $1 \leqslant u \leqslant 2$. Recursively we get the result for the general case $1 \leqslant u \leqslant U$.

\section{Proof of Lemma 3.2}

Lastly we complete the postponed proof of Lemma 3.2, and there are two assertions.

5.1. Proof of (3.9). According to the definition of $h_{N_{f}, y}$ after (3.6), we have

$$
\sum_{n \leqslant y^{u}} h_{N_{f}, y}(n)=\sum_{\substack{n \leqslant y^{u} \\ P(n) \leqslant \sqrt{y}}} h_{N_{f}, y}(n)-\sum_{\substack{y<p \leqslant y^{u} \\ p \nmid N_{f}}} \sum_{n \leqslant y^{u} / p} h_{N_{f}, y}(n)
$$

for all $u$ and $y$ satisfying (3.10).

With the Buchstab identity, it follows that

$$
\sum_{\substack{n \leqslant y^{u} \\ P(n) \leqslant \sqrt{y}}} h_{N_{f}, y}(n)=\Xi_{N_{f}, 2}\left(y^{u}, y^{1 / 4}\right)+\left(\sum_{\substack{y^{1 / 4}<p \leqslant y^{1 / 3} \\ p \nmid N_{f}}} \kappa_{3}+\sum_{\substack{y^{1 / 3}<p \leqslant y^{1 / 2} \\ p \nmid N_{f}}}\right) \sum_{\substack{n \leqslant y^{u} / p \\ P(n)<p}} h_{N_{f}, y}(n) .
$$

Repeating this procedure, we obtain

$$
\sum_{\substack{n \leqslant y^{u} \\ P(n) \leqslant \sqrt{y}}} h_{N_{f}, y}(n) \geqslant S_{1}+S_{2}+S_{3},
$$


where

$$
\begin{aligned}
S_{1} & :=\Xi_{N_{f}, 2}\left(y^{u}, y^{1 / 4}\right), \\
S_{2} & :=\left(\sum_{\substack{y^{1 / 4}<p \leqslant y^{1 / 3} \\
p \nmid N_{f}}} \kappa_{3}+\sum_{\substack{y^{1 / 3}<p \leqslant y^{1 / 2} \\
p \nmid N_{f}}}\right) \Xi_{N_{f}, 2}\left(\frac{y^{u}}{p}, y^{1 / 4}\right), \\
S_{3} & :=\left(\sum_{\substack{1 / 4 \\
y^{1 / 4}<p_{1}<p_{2} \leqslant y^{1 / 3} \\
p_{i} \nmid N_{f}}} \kappa_{3}^{2}+\sum_{y^{1 / 4}<p_{1} \leqslant y^{1 / 3}<p_{2} \leqslant y^{1 / 2}} \kappa_{p_{i} \nmid N_{f}}+\sum_{\substack{y^{1 / 3}<p_{1}<p_{2} \leqslant y^{1 / 2} \\
p_{i} \nmid N_{f}}}\right) \Xi_{N_{f}, 2}\left(\frac{y^{u}}{p_{1} p_{2}}, y^{1 / 4}\right) .
\end{aligned}
$$

In view of $(3.2)$, we have

$$
L_{N_{f}}=\log \left(\omega\left(N_{f}\right)+3\right) \ll \log _{2}(k N) \ll \log _{2} y,
$$

since $y \geqslant\left(k^{3} N^{2}\right)^{1 / 100}$. Thus $y \geqslant \exp \left(C L_{N_{f}}^{2 \mathrm{e}+2}\right)$ provided $k^{3} N^{2}$ is suitably large. So we can apply Lemma 4.2 with $q=N_{f}$ and $\kappa=2$ to write

$$
S_{1}=\rho_{2}(4 u) C_{N_{f}}(y, u),
$$

where

$$
C_{N_{f}}(y, u):=\Pi_{N_{f}, 2} y^{u}\left(\log y^{1 / 4}\right)\left\{1+O\left(\frac{\left(\log _{2} y\right)^{5}}{\log y}\right)\right\} .
$$

Similarly, by Lemma 4.2 with $q=N_{f}$, we have

$$
S_{2}=C_{N_{f}}(y, u)\left(\sum_{\substack{1 / 4<p \leqslant y^{1 / 3} \\ p \nmid N_{f}}} \kappa_{3}+\sum_{\substack{y^{1 / 3}<p \leqslant y^{1 / 2} \\ p \nmid N_{f}}}\right) \frac{1}{p} \rho_{2}\left(\frac{\log \left(y^{u} / p\right)}{\log y^{1 / 4}}\right) .
$$

Integration by parts with the prime number theorem yields

$$
\left(\sum_{y^{1 / 4}<p \leqslant y^{1 / 3}} \kappa_{3}+\sum_{y^{1 / 3}<p \leqslant y^{1 / 2}}\right) \frac{1}{p} \rho_{2}\left(\frac{\log \left(y^{u} / p\right)}{\log y^{1 / 4}}\right)=\delta_{2}(u)\left\{1+O\left(\frac{1}{\log y}\right)\right\} .
$$

Trivially we have the estimate

$$
\sum_{\substack{1 / 4 \\ y^{1 / N_{f} \leqslant y^{1 / 2}}}} \frac{1}{p} \rho_{2}\left(\frac{\log \left(y^{u} / p\right)}{\log y^{1 / 4}}\right) \ll \frac{\log (k N)}{y^{1 / 4}} \ll \frac{\left(\log _{2} y\right)^{5}}{\log y},
$$

and in summary,

$$
S_{2}=\delta_{2}(u) C_{N_{f}}(y, u)
$$

Similarly we prove that

$$
S_{3}=\delta_{3}(u) C_{N_{f}}(y, u)
$$


The treatment of the double sum in (5.1) is even simplier. For $u, y$ verifying (3.10), we have

$$
\begin{aligned}
\sum_{y<p \leqslant y^{u}} \sum_{n \leqslant y^{u} / p} h_{N_{f}, y}(n) \leqslant \sum_{y<p \leqslant y^{u}} \Xi_{N_{f}, 2}\left(\frac{y^{u}}{p}, y^{1 / 4}\right) \\
+\left(\sum_{\substack{y<p_{1} \leqslant y^{u-1 / 3} \\
y^{1 / 4}<p_{2} \leqslant y^{1 / 3}}} \kappa_{3}+\sum_{\substack{y<p_{1} \leqslant y^{u-1 / 3} \\
y^{1 / 3}<p_{2} \leqslant y^{u} / p_{1}}}+\sum_{\substack{y^{u-1 / 3}<p_{1} \leqslant y^{u-1 / 4} \\
y^{1 / 4}<p_{2} \leqslant y^{u} / p_{1}}} \kappa_{3}\right) \Xi_{N_{f}, 2}\left(\frac{y^{u}}{p_{1} p_{2}}\right) .
\end{aligned}
$$

The previous argument applies and we get that

$$
\sum_{y<p \leqslant y^{u}} \sum_{n \leqslant y^{u} / p} h_{N_{f}, y}(n) \leqslant \delta_{4}(u) C_{N_{f}}(y, u) .
$$

Inserting (5.2), (5.3), (5.4) and (5.5) into (5.1), we get the desired inequality in $(3.9)$.

5.2. Study of $\delta(u)$. To facilitate the numerical computation, we put

$$
v:=4 u, \quad \tilde{\delta}_{i}(v):=\delta_{i}(v / 4) \quad \text { and } \quad \tilde{\delta}(v)=\delta(v / 4) .
$$

Thus we have

$$
\tilde{\delta}(v)=\tilde{\delta}_{1}(v)+\tilde{\delta}_{2}(v)+\tilde{\delta}_{3}(v)-\tilde{\delta}_{4}(v) \quad(16 / 3 \leqslant v \leqslant 6) .
$$

After some standard manipulations with the change of variables, the interchange of integrals and integration by parts, we deduce that

$$
\begin{aligned}
\tilde{\delta}_{1}(v)= & \rho_{2}(v), \\
\tilde{\delta}_{2}(v)= & \int_{v-2}^{v-4 / 3} \frac{\rho_{2}(t)}{v-t} \mathrm{~d} t+\kappa_{3} \int_{v-4 / 3}^{v-1} \frac{\rho_{2}(t)}{v-t} \mathrm{~d} t, \\
\tilde{\delta}_{3}(v)= & \int_{v-4}^{v-10 / 3} \frac{\rho_{2}(t)}{v-t} \log \left(\frac{2}{v-2-t}\right) \mathrm{d} t+\int_{v-3}^{v-2} \frac{\rho_{2}(t)}{v-t} \log (v-1-t) \mathrm{d} t \\
& +\int_{v-10 / 3}^{v-3} \frac{\rho_{2}(t)}{v-t}\left\{\kappa_{3} \log \left(\frac{2}{v-2-t} \frac{4 / 3}{v-4 / 3-t}\right)+\log \left(\frac{v-4 / 3-t}{4 / 3}\right)\right\} \mathrm{d} t \\
& +\left(\kappa_{3}^{2}-1\right) \int_{v-8 / 3}^{v-7 / 3} \frac{\rho_{2}(t)}{v-t} \log \left(\frac{4 / 3}{v-4 / 3-t}\right) \mathrm{d} t, \\
\tilde{\delta}_{4}(v)= & v \log \left(\frac{v}{v-1}\right)-1+\int_{1}^{4 / 3} \frac{\left(3-\kappa_{3}\right) t-\left(2-\kappa_{3}\right) t \log t-\left(2-\kappa_{3}\right)}{v-t} \mathrm{~d} t \\
& +\int_{4 / 3}^{v-4} \frac{\left(2+\left(\kappa_{3}-1\right) \log (4 / 3)\right) t-t \log t-\left(2+\kappa_{3}\right) / 3}{v-t} \mathrm{~d} t .
\end{aligned}
$$

Next we show that all summands on right-hand side of $\tilde{\delta}_{i}(v)(1 \leqslant i \leqslant 3)$ are decreasing on $[4+\sqrt{\mathrm{e}}, 6]$. The proofs are quite similar, so we only consider, as an example, the third summand in the expression of $\tilde{\delta}_{3}(v)$. Denote this term by $\tilde{\delta}_{3,3}(v)$ and define

$$
F_{3,3}(t, v):=\frac{1}{v-t}\left\{\kappa_{3} \log \left(\frac{2}{v-2-t} \frac{4 / 3}{v-4 / 3-t}\right)+\log \left(\frac{v-4 / 3-t}{4 / 3}\right)\right\} .
$$


Noticing

we infer that

$$
\frac{\partial F_{3,3}}{\partial v}(t, v)=-\frac{\partial F_{3,3}}{\partial t}(t, v)
$$

$$
\begin{aligned}
\tilde{\delta}_{3,3}^{\prime}(v) & =\left[\rho_{2}(t) F_{3,3}(t, v)\right]_{v-10 / 3}^{v-3}-\int_{v-10 / 3}^{v-3} \rho_{2}(t) \frac{\partial F_{3,3}}{\partial t}(t, v) \mathrm{d} t \\
& =\int_{v-10 / 3}^{v-3} \rho_{2}^{\prime}(t) F_{3,3}(t, v) \mathrm{d} t \\
& <0
\end{aligned}
$$

since $\rho_{2}^{\prime}(t) \leqslant 0$ for $t \geqslant \sqrt{\mathrm{e}}$ and $F_{3,3}(t, v)>0$ for $v-10 / 3 \leqslant t \leqslant v-3$.

Consequently we have

$$
\begin{aligned}
\tilde{\delta}_{4}^{\prime}(v)= & \log \left(\frac{v}{v-1}\right)+\int_{1}^{4 / 3} \frac{1-\left(2-\kappa_{3}\right) \log t}{v-t} \mathrm{~d} t \\
& +\int_{4 / 3}^{v-4} \frac{1+\left(\kappa_{3}-1\right) \log (4 / 3)-\log t}{v-t} \mathrm{~d} t \\
> & 0 .
\end{aligned}
$$

Hence $\tilde{\delta}(v)$ is decreasing on $[4+\sqrt{\mathrm{e}}, 6]$.

Using MAPLE, we check that $\tilde{\delta}(40 / 113)>0.002 \ldots$ Thus $\tilde{\delta}\left(\tilde{u}_{0}\right)=0$ with $\tilde{u}_{0}>$ $113 / 20>4+\sqrt{\mathrm{e}}$, and we have $\delta\left(u_{0}\right)=0$ with $u_{0}=\tilde{u}_{0} / 4>113 / 80$.

\section{REFERENCES}

[1] J.-H. Bruinier \& W. Kohnen, Sign changes of coefficients of half integral weight modular forms, In: Modular forms on Schiermonnikoong (eds. B. Edixhoven et. al.), 57-66, Cambridge Univ. Press, 2008.

[2] D. A. Burgess, On character sums and L-series. II, Proc. London Math. Soc. (3) 13 (1963), $524-536$.

[3] K. Chandrasekharan, Arithmetical functions, Die Grundlehren der mathematischen Wissenschaften, Band 167 Springer-Verlag, New York-Berlin (1970).

[4] R. de la Bretèche \& G. Tenenbaum, Propriétés statistiques des entiers friables, Ramanujan J. 9 (2005), 139-202.

[5] W. Duke \& E. Kowalski, A problem of Linnik for elliptic curves and mean-value estimates for automorphic representations, With an appendix by Dinakar Ramakrishnan, Invent. Math. 139 (2000), no. 1, 1-39.

[6] S. Gelbart \& H. Jacquet, A relation between automorphic representations of $G L(2)$ and GL(3), Ann. Sci. École Norm. Sup. (4) 11 (1978), 471-552.

[7] S. W. Graham \& C. J. Ringrose, Lower bounds for least quadratic nonresidues, in: Analytic number theory, edited by Bruce C. Berndt, Harold G. Diamond, H. Halberstam and A. Hildebrand, Progr. Math., Vol. 85 (Birkhäuser Boston, 1990), pp. 269-309.

[8] G. Hanrot, G. Tenenbaum \& J. Wu, Moyennes de certaines fonctions multiplicatives sur les entiers friables, 2, Proc. London Math. Soc. (3) 96 (2008), 107-135.

[9] H. Iwaniec, W. Kohnen \& J. Sengupta, The first sign change of Hecke eigenvalue, International J. Number Theory 3 (2007), no. 3, 355-363.

[10] W. Kohnen, Y.-K. Lau \& J. Wu, Fourier coefficients of cusp forms of half-integral weight, Preprint 2009. 
[11] W. Kohnen \& J. Sengupta, On the first sign change of Hecke eigenvalues of newforms, Math. Z. 254 (2006), no. 1, 173-184.

[12] E. Kowalski, Excluding certain bad behavior of Fourier coefficients of modular forms, preprint 2007, http://www.math.u-bordeaux1.fr/ kowalski/notes-unpublished.html

[13] E. Kowalski, Y.-K. Lau, K. Soundararajan \& J. Wu, On modular signs, Math. Proc. Camb. Phil. Soc. 149 (2010), 389-411.

[14] E. Kowalski, P. Michel \& J. VanderKam, Rankin-Selberg L-functions in the level aspect, Duke Math. J. 114 (2002), no. 1, 123-191.

[15] Y.-K. Lau, J.-Y. Liu \& J. Wu, Coefficients of symmetric square L-functions, Science in China Series A: Mathematics 53 (2010), No. 9, 2317-2328.

[16] Y.-K. Lau \& J. Wu, On the least quadratic non-residue, International J. Number Theory 4 (2008), no. 3, 423-435.

[17] Y.-K. Lau \& J. Wu, A large sieve inequality of Elliott-Montgomery-Vaughan type and two applications, IMRN, Vol. 2008, Number 5, Article ID rnm 162, 35 pages.

[18] Yu.V. Linnik, A remark on the least quadratic non-residue, C. R. (Doklady) Acad. Sci. URSS (N.S.) 36 (1942), 119-120.

[19] J.-Y. Liu, Y. Qu \& J. Wu, Two Linnik-type problems for automorphic L-functions, Preprint 2010.

[20] K. Matomäki, On signs of Fourier coeffcients of cusp forms, Preprint 2010.

[21] H. L. Montgomery \& R. C. Vaughan, Extreme values of Dirichlet L-functions at 1, in: Number theory in progress, Vol. 2, Zakopane-Kościelisko, 1997 (K. Györy, H. Iwaniec \& J. Urbanowicz, Eds), 1039-1052, de Gruyter, Berlin, 1999.

[22] Y. Qu, Linnik-type problems for automorphic L-functions, J. Number Theory 130 (2010), no. $3,786-802$.

[23] J.-P. Serre, Quelques applications du théorème de densité de Chebotarev, Inst. Hautes Études Sci. Publ. Math. 54 (1981), 323-401.

[24] G. Shimura, On the holomorphy of certain Dirichlet series, Proc. London Math. Soc. 31 (1975), 79-98.

[25] G. Shimura, Introduction to the arithmetic theory of automorphic functions, Reprint of the 1971 original, Publications of the Mathematical Society of Japan, 11, Kanô Memorial Lectures, 1. Princeton University Press, Princeton, NJ, 1994, xiv+271 pp.

[26] H. Smida, Sur les puissances de convolution de la fonction de Dickman, Acta Arith. 59 (1991), 123-143.

[27] G. Tenenbaum, Introduction to analytic and probabilistic number theory, Translated from the second French edition (1995) by C. B. Thomas. Cambridge Studies in Advanced Mathematics, 46. Cambridge University Press, Cambridge, 1995, xvi+448 pp.

[28] G. Tenenbaum \& J. Wu, Moyennes de certaines fonctions multiplicatives sur les entiers friables, J. Reine Angew. Math. 564 (2003), 119-167.

[29] G. Tenenbaum \& J. Wu, Moyennes de certaines fonctions multiplicatives sur les entiers friables, 3, Compositio Math. 144 (2008), No. 2, 339-376.

[30] I. M. Vinogradov, Sur la distribution des résidus et non résidus de puissances, Permski J. Phys. Isp. Ob. -wa 1 (1918), 18-28 and 94-98.

Department of Mathematics, The University of Hong Kong, Pokfulam Road, HONG KONG

E-mail address: yklau@maths.hku.hk

School of Mathematics, Shandong University, Jinan, Shandong 250100, China

E-mail address: jyliu@sdu.edu.cn

Institut Elie Cartan Nancy (IECN), NanCy-Université CNRS INRIA, Boulevard des Aiguillettes, B.P. 239, 54506 Vanderuvre-Lès-Nancy, France

E-mail address: wujie@iecn.u-nancy.fr 